\section{Saúde mental e a qualidade organizacional dos serviços de atenção primária no Brasil}

\author{
Mental health and organizational quality of \\ primary healthcare services in Brazil
}

\section{Salud mental y la calidad organizativa de los servicios de atención primaria en Brasil}

Patricia Rodrigues Sanine 1

Letícia Isabel Ferreira Silva 1

doi: 10.1590/0102-311X00267720

\section{Resumo}

Objetivou-se avaliar a qualidade das práticas de atenção às pessoas com sofrimento relacionado à saúde mental nos serviços de atenção primária à saúde do Brasil e sua associação com variáveis de estrutura organizacional por meio de pesquisa avaliativa com dados do Programa Nacional de Melhoria do Acesso e da Qualidade da Atenção Básica (PMAQ-AB), coletados em 2018. Foram excluídas as equipes que informaram não realizar esse tipo de cuidado. A soma dos 13 indicadores de saúde mental selecionados gerou um escore que foi distribuído em três grupos de qualidade: G1 ( 0 a 5 pontos - menor qualidade), G2 (6 a 9 pontos - qualidade mediana) e G3 (10 a 13 pontos - maior qualidade), e seus resultados foram associados a nove variáveis de contexto organizacional favorecedoras à permanência das equipes nos respectivos grupos de qualidade. Foram avaliadas 36.384 equipes, localizadas em $5.026 \mathrm{mu}$ nicípios. O escore variou entre 1 e 13 pontos $(G 1=9,7 \%$; $G 2=25,1 \%$ e $G 3=$ 65,2\%). Ter ambiente que propicie dispensação de medicamento e privacidade na consulta, existência de médico especialista, psicólogo e farmacêutico, apoio matricial pelo Núcleo Ampliado de Saúde da Família (NASF) e Centro de Atenção Psicossocial (CAPS), além de reuniões de equipe semanal elou quinzenal, estiveram presentes em maior número entre as equipes que compuseram o grupo de maior qualidade $(p<0,001)$. Concluiu-se que condições estruturais relacionadas à infraestrutura e à disponibilidade de profissionais, assim como apoio matricial e espaços de reflexão para discutir a (re)organização dos processos de trabalho na atenção aos usuários com sofrimento relacionado à saúde mental são fatores que influenciam a qualidade da atenção, precisando ser considerados, especialmente, diante dos retrocessos politicos vivenciados.

Avaliação em Saúde; Saúde Mental; Atenção Primária à Saúde; Serviços de Saúde; Gestão em Saúde

\author{
Correspondência \\ P. R. Sanine \\ Rua Carlos Guadanini 2406, Botucatu, SP 18610-120, Brasil. \\ patsanine@yahoo.com.br \\ 1 Faculdade de Medicina, Universidade Estadual Paulista, \\ Botucatu, Brasil.
}




\section{Introdução}

No Brasil, a saúde mental carrega uma enorme carga de doença, destacando a depressão e a ansiedade como principais causas de anos de vida perdidos por morte ou incapacidade, entre 1990 e 20151.

Estudos que comprovam maior risco de adoecimento mental entre expostos às condições sociais, econômicas e ambientais não favoráveis 2 reforçam um cenário preocupante, incluindo o aumento das taxas de suicídio em decorrência de implantação de medidas de austeridade como resposta a crises econômicas ${ }^{3}$. Considerando que o Brasil evidencia a incorporação de políticas desfavoráveis aos programas sociais 4,5 , agravados pela pandemia da COVID-196, estima-se uma tendência de piora desse quadro.

Especialmente em relação à pandemia, apesar das contradições presentes no país - que incentiva a circulação de bens e pessoas, mesmo diante do adoecimento e morte -, os impactos negativos desse momento para a economia são inquestionáveis, assim como o empobrecimento da população e a desestruturação dos serviços de saúde, que já vinham sofrendo com o (des)financiamento operacionalizado por este modelo neoliberal de gestão pública 4,5,6.

Soma-se a esse contexto, os reflexos diretos, e indiretos, relacionados à saúde mental, como o aumento da ansiedade em decorrência do isolamento social, da insegurança financeira e do risco em se infectar, além da propensão à descontinuidade do tratamento, mudanças nos hábitos de vida, entre outros $7,8,9$.

Assim, o impacto que esses agravos causam às pessoas com sofrimento relacionado à saúde mental e a seus familiares é refletido na sociedade como um todo, representando grande parte dos gastos com a saúde - individual e pública 3 .

Buscando reduzir tais impactos, organizações nacionais e internacionais recomendam a incorporação de ações relacionadas à saúde mental na rotina dos serviços de atenção primária à saúde (APS) e sua integração com os demais níveis de atenção 10,11.

Ao considerar a interferência dos condicionantes sociais sobre a saúde mental, há de se reconhecer a potencialidade desses serviços que, pela proximidade com a comunidade, apresentam-se como o nível de atenção com maior possibilidade de construção de vínculo, acompanhamento longitudinal e, portanto, mais aptos a identificar manifestações simples da doença, atuando na prevenção do seu agravo $2,11,12$.

O Brasil, pelo menos desde 2010, corrobora com essa proposição apostando em um modelo organizado em rede, que busca integrar diferentes serviços por meio da coordenação dos serviços de APS $13,14,15,16$.

Entre suas cinco redes prioritárias, estipula-se a Rede de Atenção Psicossocial (RAPS) como uma das linhas de cuidado estruturadas para o enfrentamento dos problemas de saúde mental. Por meio da atuação interdisciplinar dos Centros de Atenção Psicossocial (CAPS) e da inserção do psicólogo junto aos Núcleos de Apoio à Saúde da Família (NASF), busca maior integração no cuidado e redução da institucionalização desse grupo populacional 12,13,16,17.

Entretanto, apesar da ampliação dessas estratégias de enfrentamento, observa-se a manutenção de um modelo de atenção fragmentado e que, ainda, alimenta o "estigma da loucura", dificultando o acesso desses usuários aos serviços de APS 12,18.

Reconhecendo que é no cotidiano dos serviços de APS que ocorre a maior parte da produção da saúde, em seu conceito mais amplo de promoção da saúde e prevenção de agravos 19, reforça-se a necessidade de serviços bem estruturados, que avancem para além do simples diagnóstico e encaminhamento para a atenção especializada.

Partindo desse princípio e do questionamento de como estão estruturados esses serviços no país, o presente trabalho teve como objetivo avaliar a qualidade das práticas de atenção às pessoas com sofrimento relacionado à saúde mental dos serviços de APS no Brasil e sua associação com variáveis de estrutura organizacional. 


\section{Método}

Foi realizada pesquisa avaliativa, de corte transversal, que utilizou o referencial teórico de Donabedian 20 para análise dos dados da avaliação externa do 3o ciclo do Programa Nacional de Melhoria do Acesso e da Qualidade da Atenção Básica (PMAQ-AB) em todo o território nacional.

$\mathrm{O} P M A Q-A B$ visa incentivar os gestores municipais e as equipes de APS a melhorar a qualidade dos serviços por meio de ciclos avaliativos compostos pelas etapas de adesão e pactuação dos compromissos, autoavaliação e avaliação externa, encerrando com a certificação das equipes, que passam a receber um incentivo financeiro (conforme o grau de desempenho alcançado) (Ministério da Saúde. PMAQ-AB. http://aps.saude.gov.br/ape/pmaq/ciclo3/, acessado em 28/Jan/2020).

O 3o ciclo avaliativo foi inicialmente ofertado a todos os serviços de APS do país que estavam com o Cadastro Nacional de Estabelecimentos de Saúde (CNES) ativo, entre julho e setembro de 2015, e que atenderam aos critérios preconizados na Política Nacional de Atenção Básica (PNAB), especificamente em relação à carga horária dos profissionais (médicos e enfermeiros).

Os dados foram coletados, durante o ano de 2018 (PMAQ-AB http://aps.saude.gov.br/ape/pmaq/ ciclo3/, acessado em 28/Jan/2020), por entrevistadores treinados que utilizaram um instrumento de perguntas fechadas sobre a estrutura dos serviços e organização de todo o processo de trabalho na APS, dividido em seis módulos. Esses módulos e o banco original encontram-se disponíveis no site: http://aps.saude.gov.br/ape/pmaq/ciclo3/.

Para evitar um viés de seleção, todas as equipes que relataram não realizar consulta de atenção à pessoa em sofrimento relacionado à saúde mental (pergunta 23.1 do módulo II) foram excluídas do banco de dados.

Considerando como pressuposto que a qualidade do resultado depende das condições de estrutura e de como essas são utilizadas no processo de trabalho em saúde 20 , partiu-se da avaliação dos indicadores de processo para analisar o quanto as condições estruturais apresentavam associação com a qualidade organizacional e, assim, permitir novas proposições em direção à melhoria.

As normas e os padrões abrangeram os critérios compreendidos nos documentos de atenção à saúde mental na APS 11,16, selecionando 22 variáveis do conjunto de informações contidas no instrumento. Dessas, 13 possuíam capacidade de mensurar a qualidade das equipes em relação à atenção à saúde mental e, portanto, foram consideradas indicadores avaliativos da qualidade referente ao processo (módulo II do instrumento); e as outras nove foram voltadas à estrutura organizacional dessas equipes (módulo I e II do instrumento). Esse detalhamento encontra-se no Quadro 1, cujas respostas foram distribuídas de modo dicotômico, em um novo banco de dados.

Assim, o conteúdo dos 13 indicadores avaliativos permitiu mensurar a qualidade da atenção ofertada pela equipe de APS às pessoas em sofrimento relacionado à saúde mental, abordando: realização de consultas (5 indicadores); atendimento em grupo (1 indicador); registro dos usuários e dos encaminhamentos (7 indicadores).

Para melhor caracterizar o universo avaliado, buscou-se evidenciar a existência de diferenças regionais, realizando uma análise descritiva desses 13 indicadores, conforme as cinco regiões brasileiras (Norte, Sul, Sudeste, Centro-oeste e Nordeste). Para essa análise, foram utilizados os testes de qui-quadrado, executando testes $\mathrm{Z}$ para proporções, quando estatisticamente significativos $(\mathrm{p}<0,05)$.

Essas diferenças auxiliaram elucidar as práticas ofertadas pelas equipes no país, mas cabe destacar que não foram consideradas para a avaliação da qualidade das equipes, uma vez que as diretrizes organizacionais são vigentes para todo o nível nacional, e, portanto, a avaliação foi considerada para o país como um todo.

A avaliação foi realizada por meio da construção de três grupos de qualidade, calculados a partir da pontuação máxima de cada equipe de APS. Como a avaliação do PMAQ-AB tem seu foco no acesso e na qualidade do conjunto de atividades preconizadas para os serviços de APS, classificando apenas alguns dos indicadores em padrões essenciais, estratégicos, gerais e obrigatórios, não contempla nenhum dos indicadores específicos para a avaliação da pessoa em sofrimento relacionado à saúde mental (http://aps.saude.gov.br/ape/pmaq/ciclo3/, acessado em 28/Jan/2020).

Dessa forma, tendo em vista a inexistência de estudos classificatórios de tais indicadores e a preconização de todos eles pelos documentos da área 11,16, optou-se por não realizar diferenciação em seus 
Quadro 1

Seleção dos indicadores de atenção à pessoa em sofrimento relacionado à saúde mental e variáveis de contexto gerencial. Programa Nacional de Melhoria do Acesso e da Qualidade da Atenção Básica (PMAQ-AB), 2018.

\begin{tabular}{|c|c|}
\hline INDICADORES/VARIÁVEIS & QUESTÃO/ALTERNATIVA \\
\hline \multicolumn{2}{|l|}{ Atenção à pessoa em sofrimento relacionado à saúde mental (13 indicadores) } \\
\hline Realiza consulta para usuários em sofrimento mental. & II.23.1(1) \\
\hline Realiza consulta para usuários de crack, álcool e outras drogas. & II.23.1(2) \\
\hline $\begin{array}{l}\text { Realiza consulta para usuários em uso crônico de benzodiazepínicos, antipsicóticos, anticonvulsivantes, } \\
\text { antidepressivos e estabilizadores de humor. }\end{array}$ & II.23.1(3) \\
\hline Possui registro dos usuários em sofrimento mental. & II.23.2(1) \\
\hline Possui registro dos usuários de crack, álcool e outras drogas. & $11.23 .2(2)$ \\
\hline $\begin{array}{l}\text { Possui registro dos usuários em uso crônico de benzodiazepínicos, antipsicóticos, anticonvulsivantes, } \\
\text { antidepressivos e estabilizadores de humor. }\end{array}$ & II.23.2(3) \\
\hline Consulta específica com tempo maior para pessoas em sofrimento mental. & II.23.3(1) \\
\hline Registro da história de vida para pessoas em sofrimento mental. & $11.23 .3(2)$ \\
\hline Oferta de atendimento em grupo para pessoas em sofrimento mental. & $11.23 .3(3)$ \\
\hline Atendimento com profissionais de saúde mental para pessoas em sofrimento mental. & $11.23 .3(4)$ \\
\hline Possui registro dos encaminhamentos de usuários em sofrimento mental. & II.23.4(1) \\
\hline Possui registro dos encaminhamentos de usuários de crack, álcool e outras drogas. & $11.23 .4(2)$ \\
\hline $\begin{array}{l}\text { Possui registro dos encaminhamentos de usuários em uso crônico de benzodiazepínicos, antipsicóticos, } \\
\text { anticonvulsivantes, antidepressivos e estabilizadores de humor. }\end{array}$ & II.23.4(3) \\
\hline \multicolumn{2}{|l|}{ Estrutura organizacional (9 variáveis) } \\
\hline Local para dispensação de medicamento & I.6.2(1) \\
\hline Consultório permite privacidade & I.6.4(5) \\
\hline Existência de Médico especialista * & 1.3.2(1) \\
\hline Existência de Psicólogo & $1.3 .2(2)$ \\
\hline Existência de Farmacêutico & 1.3.2(6) \\
\hline Apoio matricial do NASF & II.3.2(1) \\
\hline Apoio matricial do CAPS & II.3.2(2) \\
\hline Realização de reunião semanal e/ou quinzenal & II.8.Reunião \\
\hline Participação em educação permanente & II.4.EdPermanente \\
\hline
\end{tabular}

CAPS: Centros de Atenção Psicossocial; NASF: Núcleos de Apoio à Saúde da Família.

Fonte: Ministério da Saúde (PMAQ-AB - Programa Nacional de Melhoria do Acesso e da Qualidade da Atenção Básica. http://aps.saude.gov.br/ape/ pmaq/ciclo3/, acessado em 28/Jan/2020).

Nota: II.4.EdPermanente = soma das variáveis II.4.1(1) + II.4.1(2) + II.4.1(3) + II.4.1(4) + II.4.1(5); II.8.Reunião = soma das variáveis II.8.2(1) + II.8.2(2).

* Especialidade não definida.

valores, atribuindo 1 (um) ponto para cada um dos 13 indicadores, quando presente na rotina das equipes, e 0 (zero), quando ausente. Assim, a soma da pontuação total dos indicadores variou entre 0 e 13 .

Após essa classificação, as equipes foram separadas em seus respectivos grupos de qualidade, conforme a pontuação: Grupo 1 ( 0 a 5 pontos - menor qualidade); Grupo 2 (6 a 9 pontos - qualidade mediana) e Grupo 3 (10 a 13 pontos - maior qualidade).

Para identificar as variáveis de contexto organizacional favorecedoras à permanência das equipes de APS nos respectivos grupos de qualidade, esses grupos foram associados a nove variáveis voltadas à organização geral dos serviços, ou seja: em relação à estrutura física adequada (2 variáveis); a existência de profissionais especializados na composição da equipe (3 variáveis); a presença de uma rede de apoio matricial (2 variáveis); além da periodicidade na realização da reunião de equipe (1 variável) e participação em ações de educação permanente (1 variável). 
Essas associações foram realizadas por meio da distribuição das frequências das variáveis, por testes do qui-quadrado. Para identificar as diferenças das proporções entre os grupos de qualidade, aplicaram-se testes $\mathrm{Z}$ para proporções nas estimativas estatisticamente significativas $(\mathrm{p} \leq 0,05)$.

\section{Resultados}

\section{Caracterização do universo}

Aderiram ao 3o ciclo do PMAQ-AB 38.864 equipes de APS (97\% do total das equipes do país), localizadas em 5.324 municípios dos 26 estados brasileiros e Distrito Federal, no entanto, 1.514 delas não completaram o processo avaliativo. Das 37.350 equipes homologadas para a avaliação, 966 (2,5\%) foram excluídas por informar que não realizavam nenhum tipo de consulta de atenção à pessoa em sofrimento mental, 57 (2,1\%) da Região Centro-oeste, 60 (1\%) da Sul, 230 (7,5\%) da Norte, 162 (1,3\%) da Sudeste e 457 (3,3\%) da Nordeste.

Assim, compuseram o universo de estudo 36.384 equipes de APS, localizadas em 5.026 municípios, que representam 97,4\% das equipes avaliadas em todo o Brasil (PMAQ-AB. http://aps.saude. gov.br/ape/pmaq/ciclo3/, acessado em 28/Jan/2020); (Cadastro Nacional dos Estabelecimentos de Saúde do Brasil - CNES. http://tabnet.datasus.gov.br/cgi/tabcgi.exe?cnes/cnv/atencbr.def, acessado em 29/Mai/2020).

Entre as regiões, destacam-se a Nordeste $(n=13.379)$, seguida da Sudeste $(n=12.184)$, que foram as que tiveram um maior número de equipes avaliadas. As proporções entre as equipes avaliadas e os números de serviços de APS em cada região mantiveram-se em torno de $20 \%$, ou seja, variaram entre 67,4\% e $87,2 \%$ (http://tabnet.datasus.gov.br/cgi/tabcgi.exe?cnes/cnv/atencbr.def, acessado em 29/ $\mathrm{Mai} / 2020)$.

Conforme apresentado na Tabela 1, é importante destacar que, independentemente da região do país, nenhuma das práticas avaliadas foi realizada pela totalidade das equipes. As consultas, seguidas pelo atendimento com profissionais especializados, e o registro dos usuários em sofrimento relacionado à saúde mental foram as ações mais referidas (por mais de $80 \%$ das equipes), enquanto o registro dos encaminhamentos de usuários de substâncias psicoativas (crack, álcool e outras drogas) e o atendimento em grupo foram os menos realizados ( $56,8 \%$ e $57,0 \%$, respectivamente).

Merece destaque nessa tabela a porcentagem de $97,9 \%$ das equipes que informaram realizar consultas para usuários em uso crônico de psicofármacos (benzodiazepínicos, antipsicóticos, anticonvulsivantes, antidepressivos e estabilizadores de humor), enquanto apenas $82,4 \%$ relataram registrar esses usuários.

A distribuição dessas frequências evidenciou diferenças regionais. No entanto, algumas demonstraram maior semelhança entre as regiões Sul e Sudeste (Tabela 1).

\section{Qualidade da atenção à saúde mental e o contexto organizacional}

Os escores referentes ao desempenho das equipes, segundo os 13 indicadores de qualidade organizacional da atenção à pessoa em sofrimento relacionado à saúde mental, variaram entre 1 e 13 pontos, demonstrando que, apesar da existência de equipes com baixa pontuação, todas elas realizavam, pelo menos, uma das práticas avaliadas.

A distribuição das equipes nos grupos de qualidade foi: $\mathrm{G} 1=3.540(9,7 \%)$; G2 $=9.140(25,1 \%)$ e G3 $=23.704(65,2 \%)$. Apesar de a maioria das equipes pertencer ao grupo de qualidade com maior desempenho (G3), chama atenção a existência de quase 10\% de equipes que obtiveram, no máximo, 5 pontos (G1).

A associação desses grupos de qualidade com as variáveis de estrutura organizacional permitiu evidenciar diferenças em todos eles, com exceção da realização de educação permanente, que não apresentou associação significativa, uma vez que todas as equipes afirmaram participar da atividade (Tabela 2). 
Tabela 1

Distribuição das frequências das equipes de atenção primária à saúde (APS), segundo os indicadores de qualidade organizacional da atenção à pessoa em sofrimento relacionado à saúde mental, conforme regiões do Brasil. Programa Nacional de Melhoria do Acesso e da Qualidade da Atenção Básica (PMAQ-AB), 2018.

\begin{tabular}{|c|c|c|c|c|c|c|}
\hline \multirow[t]{3}{*}{ Indicador } & \multirow{2}{*}{\multicolumn{5}{|c|}{$\begin{array}{c}\text { Frequência das equipes de APS } \\
\text { Regiões brasileiras (\%) * }\end{array}$}} & \multirow[t]{3}{*}{ Valor de $p$ * } \\
\hline & & & & & & \\
\hline & $\begin{array}{l}\text { Centro-oeste } \\
{[n=2.580]}\end{array}$ & $\begin{array}{c}\text { Nordeste } \\
{[n=13.379]}\end{array}$ & $\begin{array}{c}\text { Norte } \\
{[n=2.828]}\end{array}$ & $\begin{array}{c}\text { Sudeste } \\
{[n=12.184]}\end{array}$ & $\begin{array}{c}\text { Sul } \\
{[n=5.413]}\end{array}$ & \\
\hline \multicolumn{7}{|l|}{ Consultas para usuários } \\
\hline Em sofrimento mental & $97,4 a$ & $98,0 b$ & $95,8 c$ & $98,9 d$ & 99,5 e & $<0,001$ \\
\hline De substâncias psicoativas & $91,6 a$ & $88,0 \mathrm{~b}$ & $80,7 c$ & $95,2 d$ & $96,8 \mathrm{e}$ & $<0,001$ \\
\hline De psicofármacos & $98,0 a$ & $97,4 a$ & $93,2 b$ & $99,1 c$ & $98,9 c$ & $<0,001$ \\
\hline Com tempo maior & $71,2 a$ & $80,3 b$ & $60,6 c$ & $76,6 d$ & $75,2 e$ & $<0,001$ \\
\hline \multicolumn{7}{|l|}{ Registro dos usuários } \\
\hline Em sofrimento mental & $77,6 a$ & $84,7 b$ & $73,6 c$ & $87,0 d$ & $83,6 \mathrm{e}$ & $<0,001$ \\
\hline De substâncias psicoativas & $65,8 a$ & $69,2 b$ & $55,6 c$ & $74,6 d$ & $75,9 d$ & $<0,001$ \\
\hline De psicofármacos & $79,1 \mathrm{a}$ & $84,4 b$ & $71,1 \mathrm{c}$ & $85,6 d$ & $81,4 \mathrm{e}$ & $<0,001$ \\
\hline Registro da história de vida & $64,2 a$ & $76,3 b$ & $60,2 c$ & $74,9 d$ & $72,8 \mathrm{e}$ & $<0,001$ \\
\hline Em sofrimento mental & $63,8 a$ & $69,5 b$ & $51,2 \mathrm{c}$ & $73,8 d$ & $72,2 \mathrm{e}$ & $<0,001$ \\
\hline De substâncias psicoativas & $54,1 a$ & $53,7 a$ & $39,9 b$ & $59,2 c$ & $64,8 d$ & $<0,001$ \\
\hline De psicofármacos & $61,7 a$ & $65,6 b$ & $48,5 c$ & $67,1 d$ & $67,8 d$ & $<0,001$ \\
\hline \multicolumn{7}{|l|}{ Atendimento } \\
\hline Em grupo & $43,4 a$ & $58,4 b$ & $36,2 c$ & $59,4 b$ & $62,9 d$ & $<0,001$ \\
\hline Com profissionais especializados & $76,2 a$ & $89,1 b$ & $75,7 a$ & $91,0 c$ & $87,0 d$ & $<0,001$ \\
\hline
\end{tabular}

Fonte: Ministério da Saúde (PMAQ-AB. http://aps.saude.gov.br/ape/pmaq/ciclo3/, acessado em 28/Jan/2020).

* Testes do qui-quadrado, seguidos por testes $Z$, quando $p<0,05$. Valores seguidos da mesma letra indicam não haver diferença estatisticamente entre as proporções.

\section{Tabela 2}

Distribuição das equipes de atenção primária à saúde do Brasil, nos grupos de qualidade da atenção à pessoa em sofrimento relacionado à saúde mental e suas associações com indicadores de contexto organizacional. Programa Nacional de Melhoria do Acesso e da Qualidade da Atenção Básica (PMAQ-AB), 2018.

\begin{tabular}{|c|c|c|c|c|}
\hline \multirow[t]{4}{*}{ Indicador } & \multicolumn{3}{|c|}{ Grupos de qualidade } & \multirow[t]{4}{*}{ Valor de $p$ * } \\
\hline & $0-5$ pontos & 6-9 pontos & 10-13 pontos & \\
\hline & {$[n=3.540]$} & {$[n=9.140]$} & {$[n=23.704]$} & \\
\hline & G1 (\%) & G2 (\%) & G3 (\%) & \\
\hline Local para dispensação de medicamento & $48,8 a$ & $51,9 b$ & $58,6 c$ & $<0,001$ \\
\hline Consultório permite privacidade & $81,7 a$ & $85,9 b$ & $88,6 c$ & $<0,001$ \\
\hline Existência de médico especialista ** & $13,7 a$ & $16,8 b$ & $19,1 c$ & $<0,001$ \\
\hline Existência de psicólogo & $6,3 a$ & $8,6 b$ & $10,0 c$ & $<0,001$ \\
\hline Existência de farmacêutico & $6,1 \mathrm{a}$ & $8,1 b$ & $11,7 \mathrm{c}$ & $<0,001$ \\
\hline Apoio matricial do NASF & $59,6 a$ & $67,7 b$ & $77,3 c$ & $<0,001$ \\
\hline Apoio matricial do CAPS & $54,2 a$ & $66,5 b$ & $77,2 c$ & $<0,001$ \\
\hline Realização de reunião semanal e/ou quinzenal & $48,4 a$ & $54,0 b$ & $64,0 \mathrm{c}$ & $<0,001$ \\
\hline Participação em educação permanente & 100,0 & 100,0 & 100,0 & - \\
\hline
\end{tabular}

CAPS: Centros de Atenção Psicossocial; NASF: Núcleos de Apoio à Saúde da Família.

Fonte: Ministério da Saúde (PMAQ-AB. http://aps.saude.gov.br/ape/pmaq/ciclo3/, acessado em 28/Jan/2020).

* Testes do qui-quadrado, seguidos por testes $Z$, quando $p<0,05$. Valores seguidos da mesma letra indicam não haver diferença estatisticamente entre as proporções.

** Especialidade não definida. 
Nessa tabela, é possível observar que as equipes que afirmaram ter um ambiente para dispensação de medicamentos $(\mathrm{p}<0,001)$ e com privacidade para a realização das consultas $(\mathrm{p}<0,001)$, além da existência de médico especialista - não necessariamente em saúde mental $(\mathrm{p}<0,001)$-, psicólogo $(\mathrm{p}<$ $0,001)$ e farmacêutico $(\mathrm{p}<0,001)$, apresentaram as variáveis que estiveram presentes em maior número entre as equipes que compuseram o grupo de maior qualidade (G3). Ter apoio matricial pelo NASF $(\mathrm{p}<0,001)$ e CAPS $(\mathrm{p}<0,001)$, além da garantia de uma periodicidade (semanal e/ou quinzenal) nas reuniões de equipe $(\mathrm{p}<0,001)$, também, foram variáveis que mostraram associação com esse grupo.

\section{Discussão}

Em um país de dimensões continentais como é o caso do Brasil, a caracterização do universo avaliado torna-se mais relevante, uma vez que evidencia diversidades que precisam ser destacadas.

O número de equipes avaliadas por região é uma delas. Apesar de apontar diferenças de mais de dez mil serviços, dependendo da região, a relação entre o número de serviços e de equipes participantes manteve-se proporcional, guardando a representatividade de seus territórios.

Por outro lado, chama atenção o número de serviços que relataram não realizar atividades voltadas para pessoas em sofrimento relacionado à saúde mental, com destaque para a Região Norte, seguida da Nordeste. Tal resultado reforça as desigualdades associadas às condições socioeconômicas, historicamente existentes no país, e seus reflexos na organização dos serviços, incluindo os de APS 21,22.

No entanto, para além da localização regional dessas equipes, essa situação pode ser justificada pela falta de capacitação profissional, transversal à região. Sinaliza, ainda, um modelo de atenção que se contrapõe à lógica da reforma psiquiátrica, privilegiando a centralização dessas demandas em serviços de maior complexidade, como nas instituições asilares e/ou hospitalares. Alguns estudos sugerem coerência com esses resultados ao identificarem pouca apropriação dos profissionais de saúde em relação às questões relacionadas à saúde mental 14,23.

Apesar do já consagrado potencial dos serviços de APS como o nível de atenção com maior capacidade para o diagnóstico e acompanhamento dos casos de saúde mental, preocupa esse cenário, principalmente da região Norte, quando consideradas as carências, também, em relação aos outros níveis de atenção, como, por exemplo, dos CAPS, cuja concentração, nessa região, é a segunda com menor número de todo o país (http://tabnet.datasus.gov.br/cgi/tabcgi.exe?cnes/cnv/atencbr.def, acessado em 29/Mai/2020).

Tais resultados corroboram com estudos que demonstram uma baixa e heterogênea cobertura da RAPS, sugerindo que grande parte dessa demanda fique sem acesso, pelo menos, nos estágios iniciais da doença ou nos quadros clínicos menos severos 10,12,13,14 (http://tabnet.datasus.gov.br/cgi/tabcgi. exe?cnes/cnv/atencbr.def, acessado em 29/Mai/2020).

É importante destacar que essa abordagem, quando identificada pelos serviços de APS, tende a ocorrer em um estágio mais precoce da doença, além de possibilitar um acompanhamento prolongado, que favorece a detecção e intervenção em tempo oportuno, viabilizando melhores desfechos no momento da crise, além de redução no número de internações psiquiátricas 11.

Somam-se a essa questão, mesmo que de forma mais descritiva, o gap evidenciado entre as preposições dos documentos para a atenção ao sofrimento relacionado à saúde mental nos serviços de APS 11,16 e as práticas ofertadas pelas equipes, demonstrando o quanto, mesmo diante da existência de diretrizes que buscam avançar em uma abordagem integral e mais inclusiva desses usuários, essa, ainda, encontra-se distante das rotinas dos serviços, reproduzindo antigos modelos de atenção 12,13,24,25.

Tal comportamento pode justificar-se pela existência de tensões nas normatizações da própria RAPS, como, por exemplo, a que preconiza a inclusão das pessoas em sofrimento relacionado à saúde mental junto à comunidade, porém, ao mesmo tempo, propõe uma forma de financiamento dos serviços que valoriza a ampliação de leitos hospitalares para internações psiquiátricas em detrimento da ampliação dos CAPS e NASF 13,26,27,28,29.

Em contrapartida, mesmo diante de tais contextos, chama atenção o grande número de equipes que se concentraram no grupo de melhor qualidade (G3), sinalizando a existência de uma estrutura necessária aos serviços (profissional, infraestrutura e de equipes de apoio) e maior organização dos processos de trabalho na atenção à saúde mental ofertada pelas equipes. 
Essa interrelação entre estrutura e processo já era referenciada como condicionante para o bom resultado da qualidade 20 . No entanto, em contextos de crise política e econômica, como os vivenciados no país, as limitações estruturais tendem a ser agravadas, piorando esse cenário 2,3,4,5,6,21.

Nesse sentido, preocupa observar o comportamento de todas as variáveis de estrutura que ampliaram os grupos de melhor qualidade, conforme o aumento de sua disponibilidade nos serviços. Conhecer esse desempenho em função das condições estruturais com que as equipes podem contar é de extrema relevância para a identificação de estratégias que otimizem o melhor aproveitamento dos recursos já existentes 30,31 .

As condições de infraestrutura relacionadas à privacidade dos consultórios, além da existência de local para dispensação de medicamentos, estiveram presentes nos serviços de mais da metade das equipes entrevistadas, com maior concentração conforme melhor desempenho do grupo de qualidade. É importante destacar que tais variáveis, apesar de não se referirem especificamente à saúde mental, são condições que já comprovaram ampliar o acesso dos usuários na atenção de outros grupos específicos 32 .

No entanto, mesmo na presença de uma infraestrutura que garanta privacidade durante a consulta e favoreça o acesso a medicamentos e, consequentemente, de uma maior atenção, tais espaços não seriam suficientes diante da carência de recursos humanos qualificados para tal abordagem.

Sem aprofundar a discussão dos diferentes arranjos organizacionais sobre o modelo de atenção operacionalizado pelos serviços de APS do país, eram esperadas baixas frequências no número de equipes com profissionais especializados, uma vez que há uma certa indução, no Brasil, para a adesão de serviços do tipo saúde da família 33,34. Entretanto, assim como evidenciado no Estado de São Paulo 18, cabe ponderar que a existência desses profissionais demonstrou associação com o grupo de melhor desempenho, sugerindo que sua presença qualifica a atenção prestada à saúde mental.

Esses resultados podem sinalizar uma expansão de arranjos híbridos do modelo saúde da família, ou seja, que não atendem rigorosamente os critérios organizacionais preconizados ou mesmo a transferência desse espaço de atuação dos médicos generalistas e/ou de saúde da família para os especialistas, como um reflexo da falta crônica de médicos nos serviços de APS e do próprio movimento de desconstrução de um compromisso com a expansão da saúde da família 22,26.

Por outro lado, prenunciam uma das possíveis causas para a saúde mental não ser abordada por algumas das equipes, articulando-se com estudos que relatam a pouca apropriação dos profissionais sobre o tema $14,21,23,34$.

As equipes multidisciplinares que atuam no NASF foram criadas com a intenção de melhor instrumentalizar os profissionais da APS, ampliando a resolutividade e abrangência dos serviços, por meio do matriciamento 12. Especificamente para a saúde mental, o apoio matricial é proposto pelos CAPS, que têm se demonstrado como a melhor forma de responder a essas demandas na APS, suprindo possíveis limitações das equipes 24,34,35,36.

No entanto, é importante se atentar que o modo como o serviço de APS se organiza interfere nesse processo de matriciamento, como em relação ao profissional responsável pela saúde mental (psicólogo ou médico psiquiatra) 31.

Considerando a dimensão de suporte educacional, igualmente envolvida no apoio matricial 37, infere-se que tais discussões em conjunto com as reuniões de equipes, ou mesmo quando ocorrem somente entre os profissionais da APS, podem representar potenciais mecanismos para a qualificação da equipe, promovendo reflexões sobre os casos e a organização dos processos de trabalho 34,35,38,39.

Tal comportamento ficou evidenciado ao concentrar equipes que relataram contar com esse tipo de apoio no grupo de melhor qualidade (G3), assim como com os que informaram a realização de reuniões periódicas semanais e/ou quinzenais. Entretanto, é possível observar a falta de periodicidade para as reuniões de equipe da APS, além da existência da condução de práticas desarticuladas entre as equipes. Esses resultados são apontados em outros estudos - mesmo que nem todos diretamente voltados para esse grupo populacional 14,24,34,38,39.

Nessa perspectiva, pesa assinalar que o próprio processo avaliativo e a devolutiva de seus resultados apresentam-se como momentos que podem promover reflexões coletivas sobre o trabalho desempenhado pelas equipes, favorecendo a produção de conhecimento e a melhor qualificação dos profissionais $40,41,42$. 
Outra atividade que merece destaque como forte aliada na qualificação dos profissionais é a participação em atividades de educação permanente, oportunizando, inclusive, uma melhor compreensão sobre a integração com as equipes de matriciamento 22. Entretanto, apesar de todos os avaliados informarem participar de tais atividades, e, portanto, não demonstrando associação com o desempenho dos grupos de qualidade, estudos apontaram que essa não é a realidade em todos os locais 22,24.

Cabe pontuar que tais práticas são relativamente recentes no país, com início de investimentos para atividades formativas a partir do ano de 2005 , e que, desde então, houve um aumento no número de equipes de APS que passaram a participar de atividades de educação permanente 21,22.

Esses contextos estruturais, que demonstraram associação com os grupos de qualidade e, ao mesmo tempo, destacaram fragilidades organizacionais, ganham maior relevância quando analisados em conjunto com as atuais iniciativas governamentais. No período dessas entrevistas, já se evidenciavam movimentos de desmonte por parte do Estado, como a tendência de redução no financiamento e a centralização de práticas em alguns serviços 26,28,43.

As mudanças na política da saúde mental e no financiamento da APS abriram precedência para um movimento de maior precarização, com redução das equipes que realizavam apoio matricial (CAPS e NASF), reforçando retrocessos nos avanços já alcançados em direção ao modelo ampliado de saúde mental que se almeja - com maior equidade e menor "medicalização" -, por meio de uma abordagem integral coordenada em rede pelos serviços de APS 12,13,14,26,27,28,29.

Tais retrocessos, alinhados ao cenário de agravamento dos sofrimentos relacionados à saúde mental provocados pela pandemia da COVID-19 7,8, reforçam a necessidade de um estudo aprofundado dos serviços excluídos dessa análise por relatarem não atenderem esse grupo populacional, uma vez que, mesmo representando $2,5 \%$ do universo avaliado, sugerem maior insuficiência organizacional a um número importante de pessoas.

Ainda merece destaque uma investigação do modelo de atenção operacionalizado nesses serviços (saúde da família ou tradicional) e do perfil dos profissionais de saúde (médicos e enfermeiros responsáveis por essas equipes), uma vez que a formação específica em saúde da família, assim como a organização das práticas de saúde nessa abordagem, apresenta importantes diferenças na atuação e integração com a rede de apoio 18,44 .

Apesar de tais limitações e da reconhecida importância do tema e existência de diretrizes organizacionais que buscam um certo padrão de atenção para todo o país, as análises realizadas mostraramse sensíveis ao identificar diversidades regionais quanto à oferta de ações e carências no acompanhamento dos usuários com sofrimento relacionado à saúde mental, evidenciando deficiências de acesso e de qualidade da atenção à saúde mental.

\section{Considerações finais}

A associação das variáveis de estrutura com os grupos de melhor qualidade demonstrou a necessidade de maior sensibilização das equipes e gestores, buscando conscientizá-los sobre os impactos na atenção aos usuários com sofrimento relacionado à saúde mental, promovendo reflexões e (re)organizações dos seus processos de trabalho junto a essa temática, especialmente em relação à manutenção das equipes de apoio matricial que, na contramão das diretrizes atuais, mostraram-se importantes para qualificar a atenção prestada.

Também, pode-se constatar a influência de algumas práticas que, muitas vezes, não estão relacionadas a tais políticas ou à necessidade de maior financiamento, como a potencialidade da descentralização da dispensação de medicamento, como estratégia associada às equipes concentradas nos grupos de melhor qualidade.

Espera-se que a presente discussão fomente espaços reflexivos entre as equipes de APS de todo o país, mobilizando iniciativas que favoreçam o fortalecimento de uma atenção inclusiva e integral às pessoas com sofrimento relacionado à saúde mental nos serviços de APS de todo o país. 


\section{Colaboradores}

P. R. Sanine participou de todas as etapas, incluindo a revisão do texto final. L. I. F. Silva colaborou com a construção do banco de dados, análise e revisão do texto final.

\section{Informações adicionais}

ORCID: Patricia Rodrigues Sanine (0000-00027668-0327); Letícia Isabel Ferreira Silva (00000003-2370-103X).

\section{Agradecimentos}

Os autores agradecem aos profissionais dos serviços de atenção primária à saúde comprometidos com a qualidade do cuidado e à pesquisadora Carina Akemi Nakamura, que colaborou na seleção dos indicadores, e ao Ministério da Saúde, Núcleo de Educação em Saúde Coletiva da Universidade Federal de Minas Gerais pelo financiamento.

\section{Referências}

1. Bonadiman CSC, Passos VMA, Mooney M, Naghavi M, Melo APS. A carga dos transtornos mentais e decorrentes do uso de substâncias psicoativas no Brasil: Estudo de Carga Global de Doença, 1990 e 2015. Rev Bras Epidemiol 2017; 20 Suppl 1:191-204.

2. Mental Health Foundation. Fundamental facts about mental health 2016. https://www.men talhealth.org.uk/sites/default/files/fundamen tal-facts-about-mental-health-2016.pdf (acessado em 22/Ago/2020).

3. Schramm JMA, Paes-Souza R, Mendes LVP. Políticas de austeridade e seus impactos na saúde. http://www.cee.fiocruz.br/sites/de fault/files/1_Joyce\%20M-R\%C3\%B4mulo\%20 P-Luiz\%20V_austeridade_1.pdf (acessado em 28/Jun/2020).

4. Hone T, Mirelman AJ, Rasella D, Paes-Sousa R, Barreto ML, Rocha R, et al. Effect of economic recession and impact of health and social protection expenditures on adult mortality: a longitudinal analysis of 5565 Brazilian municipalities. Lancet Glob Health 2019; 7:e1575-83.

5. Massuda A, Hone T, Leles FAG, Castro MC, Atun R. The Brazilian health system at crossroads: progress, crisis and resilience. BMJ Glob Health 2018; 3:e000829.

6. Nunes J. A pandemia de COVID-19: securitização, crise neoliberal e a vulnerabilização global. Cad Saúde Pública 2020; 36:e00063120.

7. Johnson MC, Saletti-Cuesta L, Tumas N. Emociones, preocupaciones y reflexiones frente a la pandemia del COVID-19 en Argentina. Ciênc Saúde Colet 2020; 25 Suppl 1:2447-56.

8. Castro-de-Araujo LFS, Machado DB. Impacto do COVID-19 na saúde mental em um país de baixa e média renda. Ciênc Saúde Colet 2020; 25 Suppl 1:2457-60.

9. Werneck AO, Silva DR, Malta DC, SouzaJúnior PRB, Azevedo LO, Barros MBA, et al. Lifestyle behaviors changes during the $\mathrm{CO}$ VID-19 pandemic quarantine among 6,881 Brazilian adults with depression and 35,143 without depression. Ciênc Saúde Colet 2020; 25 Suppl 2:4151-6.

10. Frankish H, Boyce N, Horton R. Mental health for all: a global goal. Lancet 2018; 392:1493-4.

11. Departamento de Atenção Básica, Secretaria de Atenção à Saúde, Ministério da Saúde. Saúde Mental. Brasília: Ministério da Saúde; 2013. (Caderno de Atenção Básica, 34).

12. Dos-Santos EM, Soares Júnior RC, Mafra ALS, Santos GM. Produção do cuidado em saúde mental na atenção básica: revisão integrativa. Revista FUNEC Científica - Enfermagem 2018; 2:38-52.

13. Fernandes CJ, Lima AF, Oliveira PRS, Santos WS. Índice de Cobertura Assistencial da Rede de Atenção Psicossocial (iRAPS) como ferramenta de análise crítica da reforma psiquiátrica brasileira. Cad Saúde Pública 2020; 36:e00049519. 
14. Fegadolli C, Varela NMD, Carlini ELA. Uso e abuso de benzodiazepínicos na atenção primária à saúde: práticas profissionais no Brasil e em Cuba. Cad Saúde Pública 2019; 35:e00097718.

15. Viana ALd'A, Bousquat A, Melo GA, Negri Filho AD, Medina MG. Regionalização e Redes de Saúde. Ciênc Saúde Colet 2018; 23:1791-8.

16. Secretaria de Atenção à Saúde, Ministério da Saúde. Implantação das redes de atenção à saúde e outras estratégias da SAS. Brasília: Ministério da Saúde; 2014.

17. Onocko-Campos RT, Amaral CEM, Saraceno B, Oliveira BDC, Treichel CAS, Delgado PGG. Atuação dos Centros de Atenção Psicossocial em quatro centros urbanos no Brasil. Rev Panam Salud Pública 2018; 42:e113.

18. Pupo LR, Rosa TEC, Sala A, Feffermann M, Alves MCGP, Morais MLS. Saúde mental na Atenção Básica: identificação e organização do cuidado no Estado de São Paulo. Saúde Debate 2020; 44 (Esp 3):107-27.

19. Paim JS. Da teoria do processo de trabalho em saúde aos modelos de atenção. In: Ayres JR, Santos L, organizadores. Saúde, sociedade e história. São Paulo: Hucitec Editora; 2017. p. 375-92.

20. Donabedian A. Special article: The quality of care: How can it be assessed? JAMA 1988; 260:1743-8

21. Macedo JP, Abreu MM, Fontenele MG, Dimenstein $\mathrm{M}$. A regionalização da saúde mental e os novos desafios da Reforma Psiquiátrica brasileira. Saúde Soc 2017; 26:155-70.

22. Gerbaldo TB, Arruda AT, Horta BL, Garnelo L. Avaliação da organização do cuidado em saúde mental na atenção básica à saúde do Brasil. Trab Educ Saúde 2018; 16:1079-94.

23. Departamento de Atenção Básica, Secretaria de Atenção à Saúde, Ministério da Saúde. Gestão da atenção básica. 3a Ed. Brasília: Ministério da Sáude; 2015. (Retratos da Atenção Básica 2012, 2)

24. Alvarez APE, Vieira ÁCDD, Almeida FA. Núcleo de Apoio à Saúde da Família e os desafios para a saúde mental na atenção básica. Physis (Rio J.) 2019; 29:e290405.

25. Rocha HA, Santos ADF, Reis IA, Santos MADC, Cherchiglia ML. Mental health in primary care: an evaluation using the Item Response Theory. Rev Saúde Pública 2018; 52:17.

26. Morosini MVGC, Fonseca AF, Lima LD. Política Nacional de Atenção Básica 2017: retrocessos e riscos para o Sistema Único de Saúde. Saúde Debate 2018; 42:11-24.

27. Ministério da Saúde. Plano Nacional de Saúde 2020-2023. Brasília: Ministério da Saúde; 2020.

28. Coordenação-Geral de Planejamento, Subsecretaria de Planejamento e Orçamento, Secretaria Executiva, Ministério da Saúde. 3o Relatório quadrimestral de prestação de contas 2019. Brasília: Ministério da Saúde; 2020.
29. Ministério da Saúde. Nota Técnica no 3/2020-DESF/SAPS/MS. Núcleo Ampliado de Saúde da Família e Atenção Básica (NASF-AB) e Programa Previne Brasil. http:// 189.28.128.100/dab/docs/portaldab/docu mentos/NT_NASF-AB_Previne_Brasil.pdf (acessado em 30/Nov/2020).

30. Tanaka OY. Avaliação em saúde: novos tempos, novas construções. In: Tanaka OY, Ribeiro EL, Almeida CAL, organizadores. Avaliação em saúde: contribuições para incorporação no cotidiano. Rio de Janeiro: Editora Atheneu; 2017. p. 1-9.

31. Godoi LPS, Belotti L, Garcia EM, Rosa TEC, Tanaka OY. Apoio matricial como ferramenta da articulação entre atenção básica e Caps: o que os dados secundários mostram? Saúde Debate 2020; 44 (Esp 3):128-43.

32. Sanine PR, Venancio SI, Silva FLG, Aratani N, Moita MLG, Tanaka OY. Atenção ao pré-natal de gestantes de risco e fatores associados no Município de São Paulo, Brasil. Cad Saúde Pública 2019; 35:e0103118.

33. Sanine PR, Zarili TFT, Nunes LO, Dias A, Castanheira ERL. Do preconizado à prática: oito anos de desafios para a saúde da criança em serviços de atenção primária no interior de São Paulo, Brasil. Cad Saúde Pública 2018; 34:e00094417.

34. Campos RO, Gama CA, Ferrer AL, Santos DVD, Stefanello S, Trapé TL, et al. Saúde mental na atenção primária à saúde: estudo avaliativo em uma grande cidade brasileira. Ciênc Saúde Colet 2011; 16:4643-52.

35. Gryschek G, Pinto AAM. Saúde mental: como as equipes de Saúde da Família podem integrar esse cuidado na Atenção Básica? Ciênc Saúde Colet 2015; 20:3255-62.

36. Wenceslau LD, Ortega F. Saúde mental na atenção primária e saúde mental global: perspectivas internacionais e cenário brasileiro. Interface (Botucatu) 2015; 19:1121-32.

37. Saraiva SAL, Zepeda J, Liria AF. Componentes do apoio matricial e cuidados colaborativos em saúde mental: uma revisão narrativa. Ciênc Saúde Colet 2020; 25:553-65.

38. Sanine PR, Dias A, Carrapato JFL, Machado DF, Mendonça CS, Placideli N, et al. Influência da gestão municipal na organização da atenção à saúde da criança em serviços de atenção primária do interior paulista. Cad Saúde Pública 2021; 37:e00242219

39. Amaral CEM, Torrenté M de ON de, Torrenté M de, Moreira CP. Apoio matricial em Saúde Mental na atenção básica: efeitos na compreensão e manejo por parte de agentes comunitários de saúde. Interface (Botucatu) 2018; 22:801-12. 
40. Caccia Bava MCG, Hirooka LB, Braga NG, Xavier JJS, Mattos ATR, Brienza AM, et al. Avanços possíveis na qualificação da APS pelos processos devolutivos a partir do PMAQ: estudo de caso envolvendo parceria entre município e universidade. In: Akerman $\mathrm{M}$, Sanine PR, Bava MCGC, Marim FA, Louvison M, Hirooka LB, et al. organizadores. Atenção básica é o caminho! Desmontes, resistências e compromissos: contribuições das Universidades Brasileiras para a avaliação. São Paulo: Hucitec Editora; 2020. p. 125-43.

41. Moraes PND, Iguti AM. Avaliação do desempenho do trabalhador como forma peculiar de prescrição do trabalho: uma análise do PMAQ -AB. Saúde Debate 2013; 37:416-26.

42. Sanine PR, Paganini LA, Louvison M, Marim FA, Silva LIF, Wiik MCG. Para além da certificação ministerial: como gestores e profissionais de saúde podem utilizar os dados do PMAQ-AB para qualificar as linhas de cuidado. In: Akerman M, Sanine PR, Bava MCGC, Marim FA, Louvison M, Hirooka LB, et al., organizadores. Atenção básica é o caminho! Desmontes, resistências e compromissos: contribuições das Universidades Brasileiras para a avaliação. São Paulo: Hucitec Editora; 2020. p. 208-41.
43. Louvison M, Sanine PR, Moraes CK, CacciaBava MCG, Hirooka LB, Marques MCC, et al. Narrativas do campo do PMAQ-AB: caminhos e descaminhos sob o olhar dos entrevistadores. In: Akerman M, Sanine PR, Bava MCGC, Marim FA, Louvison M, Hirooka LB, et al., organizadores. Atenção básica é o caminho! Desmontes, resistências e compromissos: contribuições das Universidades Brasileiras para a avaliação. São Paulo: Hucitec Editora; 2020. p. 383-402.

44. Flôr CR, Oliveira CDL, Cardoso CS, Rabelo CF, Gontijo BL, Carvalho SF, et al. Primary health care as assessed by health professionals: comparison of the traditional model versus the Family Health Strategy. Rev Bras Epidemiol 2017; 20:714-26. 


\section{Abstract}

The study aimed to assess the quality of care for persons with mental health distress in primary healthcare services in Brazil and the association with organizational structure variables. This evaluative study used data from the Brazilian $\mathrm{Na}$ tional Program to Improve Acess and Quality in Primary Care (PMAQ-AB), collected in 2018. The study excluded teams that reported not performing this type of care. The sum of the 13 selected mental health indicators produced a score that was classified in three groups of quality: G1 (0 to 5 points lower quality), G2 (6 to 9 points - medium quality), and G3 (10 to 13 points - higher quality), and the results were associated with nine organizational context variables favoring the teams' permanence in the respective quality groups. The study evaluated 36,384 teams, located in 5,026 municipalities (counties). The score ranged from 1 to 13 points ( $G 1=9.7 \%$; $G 2=25.1 \%$; $G 3=65.2 \%$ ). Having an environment favorable to dispensing medicines and privacy during patient consultations; existence of a medical specialist, psychologist, and pharmacist; inter-consultation with the Expanded Family Health Care Centers (NASF) and Centers for Psychosocial Care (CAPS); and weekly or biweekly team meetings, were more frequent in the teams with higher quality $(p<0.001)$. In conclusion, structural conditions related to infrastructure and availability of healthcare workers, as well as inter-consultation and spaces for reflection to discuss the (re)organization of work processes in care for patients with mental health distress are factors that influence the quality of care, requiring attention, especially given recent political setbacks.

Health Evaluation; Mental Health; Primary Health Care; Health Services; Health Management

\section{Resumen}

El objetivo fue evaluar la calidad de las prácticas de atención en personas con pademicientos relacionados con la salud mental, en servicios de Atención Primaria en Salud de Brasil, y su asociación con variables de estructura organizativa. Se trata de una investigación evaluativa, con datos del Programa Nacional de Mejora del Acceso y de la Calidad de la Atención Básica (PMAQ-AB), recogidos en 2018. Se excluyeron a los equipos que informaron no realizar este tipo de cuidado. La suma de los 13 indicadores de salud mental seleccionados generó una puntuación que se distribuyó en tres grupos de calidad: G1 ( 0 a 5 puntos - calidad inferior), G2 (6 a 9 puntos - calidad media) y G3 (10 a 13 puntos - mayor calidad) y sus resultados estuvieron asociados a nueve variables de contexto organizativo, favorecedoras a la permanencia de los equipos en los respectivos grupos de calidad. Se evaluaron 36.384 equipos, localizados en 5.026 municipios. La puntuación varió entre 1 y 13 puntos $(G 1=9,7 \% ; G 2=25,1 \%$; G3 = 65,2\%). Contar con un ambiente que propicie la dispensación de medicamentos y la privacidad en la consulta; la existencia de un médico especialista, psicólogo $y$ farmacéutico, apoyo matricial por parte del Núcleos Ampliados de Salud de la Familia (NASF) y Centros de Atención Psicosocial (CAPS), además de reuniones de equipo semanales y/o quincenales, estuvieron presentes en mayor número entre los equipos que compusieron el grupo de mayor calidad $(p<0,001)$. Se concluyó que las condiciones estructurales, relacionadas con la infraestructura $y$ disponibilidad de profesionales, así como el apoyo matricial y los espacios de reflexión para discutir la (re)organización de los procesos de trabajo, en la atención a los usuarios con padecimientos relacionados con la salud mental, son factores que influencian la calidad de la atención, necesitando que se tengan en consideración, especialmente, ante los retrocesos políticos vividos.

Evaluación en Salud; Salud Mental; Atención

Primária de Salud; Servicios de Salud;

Gestión en Salud
Recebido em 11/Set/2020

Versão final reapresentada em 08/Dez/2020

Aprovado 11/Dez/2020 